\section{How Colchicine Works}

\section{by our Cell Biology Correspondent}

ANY attempt to explain how colchicine functions must account for all the diverse and seemingly unrelated effects it has on cells. For example, apart from its well known ability to block mitosis by interfering with the structure of the mitotic spindle, colchicine reversibly induces the retraction of the axopods of protozoa, disrupts myofibril morphogenesis, interferes with the deposition of cellulose fibres in plant cell walls, causes acute poisoning in mammals primarily by its toxicity to the central nervous system and, most surprising of all, relieves the pain of gout.

The important discovery, made by Borisky and Taylor ( $J$. Cell Biol., 34, 525 and $535 ; 1967$ ), that colchicine binds specifically and reversibly to a subunit protein of microtubules has led them to propose that colchicine acts by preventing the formation of microtubules-a hypothesis which provides plausible explanations of all the diverse effects of colchicine.

What Borisky and Taylor have found is that tritiated colchicine administered to HeLa and $\mathrm{KB}$ cells can be recovered, after homogenization of the cells, in a soluble fraction in which the colchicine is specifically and non-covalently bound to a protein with a sedimentation coefficient of $6 S$. A similar reversible binding, with the same equilibrium constant and kinetics, takes place in vitro when the soluble extract from the cells, both at interphase and in mitosis, is incubated with the colchicine. Furthermore, the colchicine is not chemically modified when it forms the complex.

The problem then becomes one of identifying the source of $6 S$ protein, the target site of colchicine. Two pieces of evidence show it to be a sub-unit protein of microtubules. First, a survey of the colchicine binding activity of several cell types and isolated organelles has revealed a strong positive correlation between the amount of colchicine bound and the abundance of microtubules. Mitotic cells, tritiated and flagellated cells, sperm and nerve cells all bind large amounts of colchicine and the only structures known to be common to these cells are microtubules. Slime moulds, on the other hand, have few if any microtubules and bind very little colchicine. It is interesting that, although recent evidence (see Nature, 215, 345; 1967) suggests not all microtubules are identical, they all seem to share the property of strongly binding colchicine.

Second, when isolated sea urchin mitotic apparatus is extracted at low ionic strengths, the microtubules disappear and the soluble extract contains the $6 S$ colchicine-binding protein. Borisky and Taylor conclude that the $6 S$ material is a constituent of the microtubules, and Shelanski and Taylor (J. Cell Biol., 34, 549 ; 1967) present other evidence that the microtubules of sea urchin sperm tails also contain this $6 S$ protein.

Thus Borisky and Taylor propose that colchicine acts by binding to sub-unit protein of the microtubules and preventing its polymerization into microtubules. They envisage that this polymerization is an equilibrium process, possibly nucleated by centrioles, and the equilibrium can be shifted in favour of the monomer by complexing monomers with colchicine. They are apparently in the process of testing this hypothesis directly by an in vitro experiment.
All the known effects of colchicine can be plausibly explained in terms of the hypothesis-even its toxicity to the central nervous system and its relief of gout pains. Nerve cells contain microtubules and doubtless their depolymerization would have deleterious effects. It would be of great interest to know whether neurofilament protein, as well as neurotubule protein, binds colchicine because a possible relationship between the two structures has been the source of much speculation. In gout, the colchicine may well give relief to the pain by disrupting the microtubules in leucocytes, thereby inhibiting phagocytosis and relieving the inflammation that accompanies it.

\section{Soft Rot Enzymology}

\section{by our Microbiology Correspondent}

A wELL known response of plants to microbial infection is an increase of phenol oxidase activity followed by the deposition of dark oxidation products. Phenols and their derived pigments usually suppress the growth of phytopathogens in situ and limit the area of diseased tissue. In turn, certain phytopathogens are able to counter such host defence mechanisms. The factors which determine plant resistance or microbial virulence are extremely complex and, although many attempts have been made to correlate the disease condition with microbial activities, understanding in this area remains fragmentary.

The activities of phenol oxidase, pectinase and dehydrogenases during the progress of potato soft rot have been reported recently by Lovrekovich, Lovrekovich and Stahmann (Phytopathology, 5\%, 737; 1967). High concentrations of the causative bacterium Erwinia carotovora were inoculated into healthy tubers and after 24 hours the infection locus was surrounded by a large zone of white rotted tissue. This latter zone was bounded by a narrow black margin which delimited the spread of the pathogen. Disease development was very rapid for the first day, but thereafter the rotting was contained by the host. Phenol oxidation in the rotted area was clearly inhibited by the bacteria because phenol oxidase and potential substrates were present. Furthermore, when bacteria were added to pigmented potato sap, the melanoid colour was lost. The bacterial inhibition of phenol oxidation was studied in model systems containing various preparations of the enzyme and catechol as the substrate. The bacteria were able to inhibit the oxidation only when glucose was supplied to the reaction mixture, a result suggesting that the sugar acted as a source of reducing power in the inhibition. Rotted tubers possessed very high levels of dehydrogenase activity in comparison to healthy tubers, and this activity was associated with the bacteria rather than with the host tissues. The authors conclude that the white rot can be explained in terms of the action of cell bound bacterial dehydrogenases maintaining the potato phenols in a reduced state even in the presence of active phenol oxidase. How then is the spread of the rot confined?

The black infection margin comprises macerated tissue, and Stahmann and his colleagues showed that extracellular bacterial pectinase was responsible for this development. Evidently at sites removed from the pathogen, activity of the host phenol oxidase is 